\title{
Numerical Simulations of the Pore-Scale Flow in Ceramic Open-Cell Foams
}

\author{
Jesus Nain Camacho Hernandez ${ }^{1}$, Markus Schubert ${ }^{1}$, Uwe Hampel ${ }^{1,2}$ \\ ${ }^{1}$ Helmholtz-Zentrum Dresden-Rossendorf, Institute of Fluid Dynamics \\ Bautzner Landstr. 400, 01328 Dresden, Germany \\ j.hernandez@hzdr.de; m.schubert@hzdr.de \\ ${ }^{2}$ Chair of Imaging Techniques in Energy and Process Engineering, TU Dresden \\ Technische Universität Dresden 01062 Dresden, Germany \\ u.hampel@hzdr.de
}

\section{Extended Abstract}

Porous materials are abundant in nature and in industrial applications. Their ability to allow fluids passing through them is of major importance and plays a very important role in various operations, such as hydrocarbon extraction of reservoirs in oil production [1]; or by dictating the amount of heat that can be dissipated from an electronic component using force convection (e.g. heat sinks) [2]. The key indicator of how easily fluids pass through porous materials is the "permeability", which allows describing pressure loss and flow velocity in porous materials. However, the impact of droplet impingement inside open-cell foams and the retention time of a solid or liquid disperse phase cannot be described solely by the permeability. To study these phenomena current computing resources permit pore-scale CFD simulations, which relate structure and transport properties of porous materials. Instead of using equivalent geometrical models based on simple geometries just as spheres or cylinders, the genuine geometry representation of the porous structure is required, especially since porous media usually involve significant pore structure defects and feature multiple length scales, which produce different mechanisms of interaction within the pores.

The present work investigates the performance of pore-scale numerical simulations over genuine 3D geometrical representations of ceramic foams reconstructed from X-ray micro-computed tomography $(\mu \mathrm{CT})$ scans of siliconinfiltrated silicon carbide" ( $\mathrm{SiSiC}$ ) open-cell foams with different nominal pore sizes using marching cubes algorithm [3]. To disclose the airflow velocity profile inside the foam simulations of single-phase air flow were conducted using finite volume method (FVM) for a wide range of Reynolds numbers including Reynolds-averaged Navier-Stokes

(RANS) turbulence models. Good agreement was achieved especially between the computed pressure gradient and the experimental pressure measurements, even outperforming other conventional numerical models based on average pore properties such as porosity and surface area. Further simulations were carried out using a Lagrangian approach to analyze particular effects such as rebounding of a low mass fraction of the disperse phase on the ceramic structure and deposition thresholds.

The work already done pursues to improve upon the current state-of-the-art of pore-scale simulations, providing evidence of the feasibility to perform flow simulations on reconstructed representations of ceramic porous media that considerably are able to predict experimental flow properties.

\section{References}

[1] J. R. Fanchi, in Principles of Applied Reservoir Simulation. Gulf Professional Publishing, 2018.

[2] R. Singh, A. Akbarzadeh, M. Mochizuki "Sintered porous heat sink for cooling of high-powered microprocessors for server applications," International Journal of Heat and Mass Transfer, no. 52, pp. 2289-2299, 2009.

[3] W. E. Lorensen, H. E. Cline, "Marching cubes: A high resolution 3D surface construction algorithm," in Proceedings of the 14th annual conference on Computer graphics and interactive techniques, ACM New York, NY, 1987, pp 163-169. 